\title{
Application of the Finite Differences Methods to Computation of Definite Integrals
}

\author{
${ }^{\mathrm{a}}$ MEHDIYEVA GALINA, ${ }^{\mathrm{b}}$ IBRAHIMOV VAGIF, ' IMANOVA MEHRIBAN \\ ${ }^{\mathrm{a}, \mathrm{b}, \mathrm{c}}$ Computational mathematics, Baku State University \\ ${ }^{b, c}$ Institute of Control Systems named after Academician A. Huseynov, \\ ${ }^{c}$ Executive Director, Science Development Foundation \\ under the President of the Republic of Azerbaijan \\ Baku, AZERBAIJAN \\ mehriban.imanova@sdf.gov.az, ibvag47@mail.ru
}

\begin{abstract}
It is known that one of the popular methods constructed to solve the scientific and engineering problem is the finite difference method studied beginning from the known and famous scientists as the Newton, Leibniz, Euler and etc. One of the first applications of the finite difference method is defined as the conception of the derivatives for the continuous function. These methods have been successfully used in solving of the differential equations at the present time. But here have investigated the application of the finite-difference methods to compute the definite integrals. Constructed the stable methods with the high order of accuracy which are applied to computation of the definite integrals. Also has been found some connection between the constructed multistep methods, the Gauss and Chebyshev methods. The received here methods have been applied to the computation of the double definite integral. For this aim, here have been investigated the double integrals for the degenerate functions. The advantages of these methods are illustrated by using the known model integral, which has been solved by using the stable method with the degree $p=6$.
\end{abstract}

Key-Words: - Finite Differences, Computation, Systems theory

Received: September 18, 2019. Revised: January 2, 2020. Accepted: January 20, 2020. Published: January 31, 2020.

\section{Introduction}

As is known the finding of the solution of the initialvalue problem for ODEs reduced to the finding of the solution of the integral equation with the variable bounders. And to find the numerical solution of this integral equation has been used one of the known quadrature formulas. The methods received by this way have been called as the numerical methods, which have some advantages. As is known the coefficients for these methods are fixed, therefore by using these methods it is not available to construct new algorithms with the higher order of accuracy. For example, the Adams methods. These methods can be received from the multistep methods as the partial case. But to receive the multistep methods from the Adams methods is not available. Because here considered some development of the finite difference methods and its application to the calculation of the definite integrals.

Let us consider the following definite integral:

$$
I=\int_{a}^{b} f(s) d s .
$$

Suppose that the continuous function of $f(x)$ is defined on the interval $[a, b]$ where it has the continuous derivatives up to some $p$, including. It is not difficult to understand that from the correlation of (1) one can write the following:

$$
y(x)=\int_{a}^{x} f(s) d s, \quad a \leq x \leq b .
$$

It is evident that from here one can be receive the next value $y(b)=I$. By taking into account that the function $y(x)$ is continuous on the interval $[a, b]$, receive:

$$
y^{\prime}(x)=f(x), y(a)=0, a \leq x \leq b .
$$

Thus receive that the calculation of the integral of (1) is equivalent to the finding of the value of $y(b)$. It follows from here that to find the value of $y(b)$ one can be used the solution of the initial-value problem (3). Thus has been shown that the calculation of definite integrals can be reduced to solve the initial-value problem for the ODE of the first order.

One of the popular methods for solving of the problem (3) can be written in the following form: 


$$
\sum_{i=0}^{k} \alpha_{i} y_{n+i}=h \sum_{i=0}^{k} \beta_{i} y_{n+i}^{\prime}
$$

This method in scientific literature is called as the finite difference method (see for example [1]-[3]). If in the equality (4) change $y^{\prime}(x)$ by the $f(x)$ (see problem (3)), then receive:

$$
\sum_{i=0}^{k} \alpha_{i} y_{n+i}=h \sum_{i=0}^{k} \beta_{i} f_{n+i},
$$

which is called as the multistep method with the constant coefficients. By the simple comparison receive that the methods (4) and (5) are equivalent. In these methods, the coefficients $\alpha_{i}, \beta_{i}$ $(i=0,1, \ldots, k)$ are some real numbers. Therefore by choosing these coefficients, one can construct the methods with the different properties. For example, if put $\alpha_{k} \neq 0$ and $\beta_{k}=0$, then receive the explicit methods. It follows to note that if consider the case $\alpha_{k}=0$ and $\beta_{k} \neq 0$, then from the formula (4), receive forward-jumping method. Let us note that the forward-jumping and implicit or explicit methods are separated methods and the forwardjumping (advanced) method cannot be received from the formula (4) as the partial case. Therefore the method (5) and the following method

$$
\begin{aligned}
& \sum_{i=0}^{k-m} \hat{\alpha}_{i} y_{n+i}=h \sum_{i=0}^{k} \hat{\beta}_{i} f_{n+i} \\
& \left(m>0,\left|\beta_{k-m+1}\right|+\ldots+\left|\beta_{k}\right| \neq 0\right)
\end{aligned}
$$

are the independent object for investigation. Thus receive two classes of methods. It is known that all methods have advantages and disadvantages. Therefore let us compare these methods by using the values of degree for stable and unstable method. To define the concept of stability and degree for the above-mentioned method let us consider the following definition:

Definition 1. The method (5) or (6) is called stable if the roots of the characteristic polynomials

$$
\rho(\lambda) \equiv \sum_{i=0}^{k} \alpha_{i} \lambda^{i} ; \hat{\rho}(\lambda) \equiv \sum_{i=0}^{k-m} \hat{\alpha}_{i} \lambda^{i}
$$

lies in the unit circle on the boundary of which there are no multiple roots.

Definition 2. The integer value $p$ is called the degree of the above-mentioned methods if the following holds:

$$
\begin{array}{r}
\sum_{i=0}^{k} \alpha_{i} y\left(x_{n+i}\right)- \\
=\sum_{i=0}^{k} \beta_{i} y^{\prime}\left(x_{n+i}\right)= \\
=O\left(h^{p+1}\right), h \rightarrow 0,
\end{array}
$$

$\sum_{i=0}^{k-m} \hat{\alpha}_{i} y\left(x_{n+i}\right)-h \sum_{i=0}^{k} \hat{\beta}_{i} y^{\prime}\left(x_{n+i}\right)=O\left(h^{p+1}\right), h \rightarrow 0$.

These definitions have been taken from the work [4] which has used by many authors (see for example [5]-[8]).

For the application of these methods to solving of some problems usually have imposed some conditions on the coefficients of them which at first have been proved in the work [4]. Let us suppose that the coefficients of the method (5) and (6) satisfy the following conditions:

A. The coefficients $\alpha_{i}(i=0,1, \ldots, k)$, $\hat{\alpha}_{i}(i=0,1, \ldots, k-m), \quad \beta_{i}, \hat{\beta}_{i}(i=0,1, \ldots, k)$ are some real numbers and $\alpha_{k} \neq 0, \hat{\alpha}_{k-m} \neq 0$.

B. The polynomials $\rho(\lambda), \sigma(\lambda)$ and $\hat{\rho}(\lambda)$, $\hat{\sigma}(\lambda)$ have no common factor different from constant which are defined as the following:

$$
\begin{array}{ll}
\rho(\lambda) \equiv \sum_{i=0}^{k} \alpha_{i} \lambda^{i} ; & \sigma(\lambda) \equiv \sum_{i=0}^{k} \beta_{i} \lambda^{i} ; \\
\hat{\rho}(\lambda) \equiv \sum_{i=0}^{k-m} \hat{\alpha}_{i} \lambda^{i} ; & \hat{\sigma}(\lambda) \equiv \sum_{i=0}^{k} \hat{\beta}_{i} \lambda^{i} .
\end{array}
$$

C. The conditions $\sigma(1) \neq 0 ; p \geq 1$ and $\hat{\sigma}(1) \neq 0$; $p \geq 1$ (for the method (6)) are hold.

The proving of this condition one can be found in [8].

Note that the method (5) is fully investigated in [4] and prove that if the method (5) has the degree $p$, then $p \leq 2 k$ is hold. But if the method is stable and has the degree $p$, then $p \leq 2[k / 2]+2$ and there are stable methods with the degree $p_{\max }=2[k / 2]+2$, but if $\beta_{k}=0$ and $\alpha_{k} \neq 0$ then $p \leq k$ (for stable methods).

The method (6) is fully investigated in [8] and prove that if the method (6) has the degree $p$, then $p \leq 2 k-m$. And if the method (6) is stable and has the degree $p$, then $p \leq k+m+1(k \geq 3 m, m>0)$. In the case $k=2$ the method with the degree $p=3$ can be written as:

$$
y_{n+1}=y_{n}+h\left(5 f_{n}+8 f_{n+1}-f_{n+2}\right) / 12 \text {. }
$$

As was noted above for the case $k=3$ in the class of methods (5) there are stable methods with the degree $p \leq 4$. But in this case, in this class of methods (6) there is the stable method with the degree $p=4$ which can be written as follows: $\backslash$ 


$$
\begin{gathered}
y_{n+2}=\left(8 y_{n+1}+11 y_{n}\right) / 19+ \\
+h\left(10 f_{n}+57 f_{n+1}+24 f_{n+2}-f_{n+3}\right) / 57 .
\end{gathered}
$$

This is the forward-jumping method. It is not difficult to determine that for the calculation of the value of definite integrals by using the forwardjumping method arises the necessity of calculation of function $f(x)$ at the extended interval $[a, b+\varepsilon]$, here $\varepsilon>0$ sufficiently small size. In the case when arise some difficulties related with the calculation of function $f(x)$ in outside of interval $[a, b]$ then one can be considered the construction of stable methods with the degree $p>k+2$ by using hybrid methods. It is known that the hybrid methods has constructed on the intersection of the one-step and multistep methods (see for example [9]-[12]). As was noted in [12] for construction of the stable methods with high degrees one can use the multistep and hybrid methods, which can be written as:

$$
\begin{gathered}
\sum_{i=0}^{k} \alpha_{i} y_{n+i}=h \sum_{i=0}^{k} \beta_{i} f_{n+i}+h \sum_{i=0}^{k} \gamma_{i} f_{n+i+v_{i}}, \\
\left(\left|v_{i}\right|<1 ; i=0,1, \ldots, k\right) .
\end{gathered}
$$

As is known all properties of the numerical methods depend on the values of its coefficients. Therefore, let us consider the finding of the values of coefficients for the method (9).

Therefore, let us consider determining of the values for the coefficients in the method of (9) by using the way of unknown coefficients. And for this aim, let us use the following Taylor series:

$$
\begin{aligned}
& y\left(x+t_{i} h\right)=y(x)+t_{i} h y^{\prime}(x)+\frac{\left(t_{i} h\right)^{2}}{2 !} y^{\prime \prime}(x)+\ldots .+ \\
& +\frac{\left(t_{i} h\right)^{p}}{p !} y^{(p)}(x)+O\left(h^{p+1}\right), h \rightarrow 0 .
\end{aligned}
$$

By choosing the values of the variables $t_{i}(i \geq 0)$ in the expression (10) one can apply the calculation of $y_{n+i}, y_{n+i}^{\prime}$ and $y_{n+i+v_{i}}^{\prime}(i=0,1, \ldots ., k)$. This expression let us use in the definition of the degree for the method (9) (The conception of the degree for the method (9) is defined by the same way which has used in the definition of the degree for the methods (5) and (6)). Then receive:

$$
\begin{gathered}
\sum_{i=0}^{k} \sum_{j=0}^{p}\left(\alpha_{i}\left(\frac{i h}{i !}\right)^{j} y^{(j)}(x)-h \beta_{i}\left(\frac{i h}{i !}\right)^{j} y^{(j+1)}(x)-h \gamma_{i}\left(\frac{\left(i+v_{i}\right) h}{i !}\right)^{j} y^{(j+1)}(x)\right)= \\
=O\left(h^{p+1}\right), h \rightarrow 0 .
\end{gathered}
$$

From here receive the following system of nonlinear algebraic equations:

$$
\begin{gathered}
\sum_{i=0}^{k} \alpha_{i}=0 ; \sum_{i=0}^{k}\left(\beta_{i}+\gamma_{i}\right)=\sum_{i=0}^{k} i \alpha_{i} \\
\sum_{i=0}^{k}\left(i \beta_{i}+\left(i+v_{i}\right) \gamma_{i}\right)=\sum_{i=0}^{k} \frac{i^{2}}{2 !} \alpha_{i} \\
(l+1) \sum_{i=0}^{k}\left(i^{l} \beta_{i}+\left(i+v_{i}\right)^{l} \gamma_{i}\right)= \\
=\sum_{i=0}^{k} i^{l+1} \alpha_{i} \quad(l=2,3, \ldots, p) .
\end{gathered}
$$

It follows that if the coefficients of the method (9) satisfies the conditions of (12), and then holds the equality of (11). It follows that the method (9) has the degree $p$.

Note that the system (12) in the case $v_{i}=0(i=0,1, \ldots, k)$ is linear but in the case $\left|v_{0}\right|+\left|v_{1}\right|+\ldots+\left|v_{k}\right| \neq 0$ is nonlinear. It is known that the found exact solution of the nonlinear system of algebraic equations is difficult. Therefore, usually to find the solution of this system is used the approximate methods. In the system (12) participated $4 k+4$ unknowns and $p+1$ equations. If $\gamma_{i}=0(i=0,1, \ldots, k)$ then by using the solution of the system (12) one can be constructed the methods with the degree $p \leq 2 k$. By taking into account this here investigated $p \leq 4 k+2$. In the case $k=1$ from the system (12), for $\alpha_{1}=-\alpha_{0}=1$ receive the following system of algebraic equations:

$$
\begin{gathered}
\beta_{0}+\beta_{1}+\gamma_{0}+\gamma_{1}=1, \\
\beta_{0}+l_{0}^{j} \gamma_{0}+l_{1}^{j} \gamma_{1}=1 /(j+1), \\
\left(j \leq 5 ; l_{i}=i+v_{i}(i=0,1)\right) .
\end{gathered}
$$

The method with the maximal degree constructed by the solution of the system (13) can be written as following:

$$
\begin{gathered}
y_{n+1}=y_{n}+h\left(f_{n+1}+f_{n}\right) / 12+ \\
+5 h\left(f_{n+1 / 2-\alpha}+f_{n+1 / 2+\alpha}\right) / 12, \alpha=\sqrt{5} / 10 .
\end{gathered}
$$

Let us note that if the method (9) is stable then there are methods with the degree $p=3 k+3$.

And now to compare the proposed here methods with the known methods let us apply some of the constructed here methods to calculate the model integral.

Let us apply the method (14) to solving of the following definite integrals

$$
\text { 1. } \int_{0}^{1}(\exp (m s)+\exp (-m s)) d s
$$


2. $\int_{0}^{1} \exp (m s) \cos (s) d s$

The corresponding initial-value problem for these examples can be written as the following:

1. $y^{\prime}=\exp (m x)+\exp (-m x), y(0)=0$

(exact

solution is $y(x)=\exp (m x)-\exp (-m x))$.

2. . $y^{\prime}=\exp (m x) \cos x, y(0)=0$ (exact solution is $\left.y(x)=((m \cos x+\sin x) \exp (m x)-m) /\left(m^{2}+1\right)\right)$.

Then received results have tabulated in the table 1 for step-sizes $h=0.1$ and $h=0.01$. For the comparison of received results with the known, here considered the cases $m= \pm 1$ and $m= \pm 5$.

The results received by using the methods (7), (8) and (14) for example 1 have been tabulated in the table 1 , but for example 2 in the table 2 .

Table1. The results for the step-size $h=0.01$

\begin{tabular}{|l|l|l|l|}
\hline $\begin{array}{l}\text { The values } \\
\text { of } m\end{array}$ & $\begin{array}{l}\text { Errors for } \\
\text { method (7) }\end{array}$ & $\begin{array}{l}\text { Errors for } \\
\text { method (8) }\end{array}$ & $\begin{array}{l}\text { Errors for } \\
\text { method } \\
(14)\end{array}$ \\
\hline$m=1$ & $4.56 \mathrm{E}-8$ & $2.26 \mathrm{E}-13$ & $4.44 \mathrm{E}-16$ \\
\hline$m=5$ & $7.76 \mathrm{E}-4$ & $9.89 \mathrm{E}-8$ & $1.51 \mathrm{E}-12$ \\
\hline$m=10$ & $9.52 \mathrm{E}-1$ & $5.04 \mathrm{E}-4$ & $1.45 \mathrm{E}-8$ \\
\hline
\end{tabular}

Table2. The results for the step-size $h=0.01$

\begin{tabular}{|l|l|l|l|}
\hline $\begin{array}{l}\text { The values } \\
\text { of } m\end{array}$ & $\begin{array}{l}\text { Errors for } \\
\text { method (7) }\end{array}$ & $\begin{array}{l}\text { Errors for } \\
\text { method (8) }\end{array}$ & $\begin{array}{l}\text { Errors for } \\
\text { method } \\
(14)\end{array}$ \\
\hline$m=1$ & $1.91 \mathrm{E}-7$ & $3.66 \mathrm{E}-13$ & $6.66 \mathrm{E}-16$ \\
\hline$m=5$ & $2.70 \mathrm{E}-5$ & $5.05 \mathrm{E}-9$ & $1.42 \mathrm{E}-13$ \\
\hline$m=10$ & $3.45 \mathrm{E}-2$ & $8.22 \mathrm{E}-6$ & $1.07 \mathrm{E}-10$ \\
\hline
\end{tabular}

And now let us consider the received here results to compute the double definite integrals.

\section{Application of multistep methods with constant coefficients to computation of double definite integrals}

As is known one of important problem in the classic mathematics is the computation of definite integrals with high accuracy. There are some classes of numerical methods for computation of double integrals, which are called as the cubature methods (see [19], p 668-683). And also it is known that by using cubature formulas in computation of double integrals accompanied with some difficulties. Therefore, in Applied Mathematics usually calculation of these integrals reduces to calculation of simple definite integrals. Note that one application of the double integrals related with investigation of geometrics figures. Therefore, the necessity of the calculation of double integrals can be arising in determination of the area or volume for some geometric objects. For example to find the value of volume for some object which is bounded by some function $z=f(x, y)$ with lateral the cylindrical surface with generator parallel to $z$-axis and from below by a flat figure $p$ on the $x y$-plane. For the finding of the volume of this figure one can be used the following double integral ( see for example [ 20, p.122-125]):

$$
V=\iint_{p} f(x, y) d p
$$

By using some assumption on the function $f(x, y)$ this integral can be written as:

$$
V=\int_{a}^{b} \int_{c}^{d} f(x, y) d x d y \text { or } V=\int_{a}^{b} d x \int_{c}^{d} f(x, y) d y .(16)
$$

Let us consider special case and suppose that the function $f(x, y)$ is degenerate, which can be presented in the following form:

$$
f(x, y)=a(x) b(y) \text {. }
$$

In this case, the integral (16) can be written as:

$$
V=\int_{a}^{b} a(x) d x \int_{c}^{d} b(y) d y
$$

By using above-described way one can calculate the integrals

$$
\int_{a}^{b} a(x) d x \text { and } \int_{c}^{d} b(y) d y
$$

and by taking that into account in the formula (17) receive the value of the integral (16). It is known that if the function is degenerate then that in general form can be written as:

$$
f(x, y)=\sum_{i=0}^{k} a_{i}(x) b_{i}(y) .
$$

By using this equality, the definite integral (16) can be calculated in the following form:

$$
\int_{a}^{b} d x \int_{c}^{d} f(x, y) d y=\sum_{i=0}^{k} \int_{a}^{b} a_{i}(x) d x \int_{c}^{d} b_{i}(y) d y
$$

By using the theory of approximation of a sufficiently smooth function by the degenerate function receives that the following is hold:

$\int_{a}^{b} \int_{c}^{d} f(x, y) d x d y=\sum_{i=0}^{k} \int_{a}^{b} a_{i}(x) d x \int_{c}^{d} b_{i}(y) d y+R_{n}(x, y)$.

Here the function $R_{n}(x, y)$ can be received in the results of approximation of $f(x, y)$ the following function: 


$$
\phi(x, y)=\sum_{i=0}^{k} a_{i}(x) b_{i}(y) .
$$

Note that depending on the construction of the numerical methods the value of the remainder term of $R_{n}(x, y)$ may be decreases. Taking into account these properties of numerical methods here have been constructed the methods of hybrid types. Constructed here hybrid method generalizes all known corresponding methods because that can be taken as the perspective direction in the theory of numerical methods.

It is evident that for the construction of more exact methods to compute the double definite integrals here proposed to use the formula (19) and apply the constructed methods to calculation of single definite integrals. For this aim, let us consider computing of the values of the following double integral:

$$
V=\int_{0}^{1} \int_{0}^{1} \exp (\lambda x+\lambda y) d x d y .
$$

By using the equality (18) in double integral (20) receives the following:

$$
V=\int_{0}^{1} \exp (\lambda x) d x \int_{0}^{1} \exp (\lambda y) d y .
$$

By the application of the methods from the section 1 , one can calculate the integrals

$$
\int_{0}^{1} \exp (\lambda x) d x \text { and } \int_{0}^{1} \exp (\lambda y) d y .
$$

Let us note that these integrals calculated by the same methods.

If to compute the double integral of (20) by the method (14) then receive the following result:

$$
\varepsilon=4.1 E-12 \text { for the } h=0 \text {. }
$$

Here $\varepsilon$ is the error of the method (14).

\section{Conclusion}

Conclusion. Here have described some surveys for our results, which have reported in the international conferences reduced at the University of Cambridge, Imperial College, University of Nanyang, Oxford University and etc.( see for example [13]-[18]). By comparison, of the known results with our results dedicated to compute the single definite integral receive that the way proposed in our papers is perspective. Taking into account these advantages of our methods, here have investigated multistep methods with constant coefficients using the first derivative of sought for function. For this aim, the explicit, implicit, forward-jumping (advanced) and hybrid methods are generalized and investigated. By comparison of the above-mentioned method have been demonstrated the advantages of the hybrid methods. Let us note that here have investigated the linear hybrid methods in general form therefore presented both theoretical ad practical interest. The abovedescribed way has been applied to computing of double integrals. For this aim, here proposed to use the indefinite double integrals for the calculation of which has been used the conception of degenerate function. Advantages of these methods illustrated on the concrete example.

The results, received in solving of the examples, are demonstrated the importance of the choice of the methods for solving of given problems.

\section{References:}

[1] Bahvalov N.S. Some remarks on the question of the numerical integration of differential equations by finite difference method, Dokl., 1955, №3, 805-808.

[2] Ryabenskii V.S., Filippov A.F. On the stability of difference equations. - M.: Gostekhizdat, 1956.

[3] Berezin, I.S., Zhidkov, N.P. 1962. Computing methods. M., Fizmatgiz, 639. (Russian)

[4] Dahlquist G. Convergence and stability in the numerical integration of ordinary differential equations, Math.Scand, 1956, No.4, 33-53.

[5] Henrici P. Discrete variable methods in ordinary differential equation. Wiley, New York, 1962.

[6] Babushka I., Vitasek E., Prager M. Numerical processes for solving differential equations, Mir, 1969, 368 p.

[7] Lambert, R.J. Numerical Methods for ordinary Differential Systems: the initial value problem. John Wiley and Sons Ltd, Chichester.

[8] Mehdiyeva G.Yu., Ibrahimov V.R. On the research of multi-step methods with constant coefficients. Monograph, Lambert. acad. publ., 2013.

[9] Skvortsov L.M. Explicit two-step RungeKutta methods. Math. modeling, 21, 2009 , 54-65.

[10] Butcher J.C. A modified multistep method for the numerical integration of 
ordinary differential equations. J. Assoc. Comput. Math., v.12, 1965, 124-135.

[11] Gear C.S. Hybrid methods for initial value problems in ordinary differential equations. SIAM, J. Numer. Anal. v. 2, 1965, 69-86.

[12] Mehdiyeva G., Imanova M., Ibrahimov V. (2011). Application of the hybrid methods to solving Volterra integrodifferential equations . World Academy of Science, Engineering and Technology, Paris, 1197-1201.

[13] Mehdiyeva G., Imanova M., Ibrahimov $\mathrm{V}$. On a one method for determining the maximum value of the order of the accuracy for the multistep hybrid method which have applied to solving of the Volterra integrodifferential equation of the first order AIP Conference Proceedings 1982, 020022 (2018); DOI: $10.1063 / 1.5045428$

[14] Mehdiyeva G., Imanova M., Ibrahimov V. Some Comparisons of the Methods Applied to Solving the First-Order IntegroDifferential Equations, IOP Conf. Series:

Materials Science and Engineering 423 (2018) 012155

[15] Mehdiyeva G., Imanova M., Ibrahimov $\mathrm{V}$. The application of the hybrid method to solving the Volterra integro-differential equation World Congress on Engineering, London U.K. 3-5 July 2013

[16] Mehdiyeva G., Ibrahimov V., Imanova M. On a calculation of definite integrals by using of the calcuilation of indefinite integrals SN Applied Sciences Springer 2019

[17] Mehdiyeva G., Ibrahimov V., Imanova M. On the comparison of the implicit advanced and hybrid methods and their application to solving Volterra inteqrodifferential equations International Journal Of Education And Information Technologies Volume 13, 2019 ISSN: 2074-1316

[18] Mehdiyeva G., Ibrahimov V., Imanova M. A Way for Finding the Relation Between of the Degree and Order for Multistep Method Used to Applied to Solving of the Volterra Integro-Differential Equation WSEAS Transactions on Mathematics Volume 17, 2018
[19] Sobolev S.L., Introduction to the theory of cubature formulas, "Nauka», Moscow 1974, 808.

[20] Fichtengolts, G.M. 1970. Course of differential and integral calculus, Volume 3, «Nauka», Moscow, 1969, 656

[21] I. G. Burova, E. F. Muzafarova , Interval Estimation using Integro-Differential Splines of the Third Order of Approximation, WSEAS Transactions on Mathematics, Volume 18, 2019, pp. 153160

[22] Jacob Manale, Integrating the Gaussian through Differentiable Topological Manifolds, WSEAS Transactions on Mathematics, Volume 18, 2019, pp. 55-61 